\title{
SMAFramework: Arcabouço para Integração de Dados Urbanos Cientes da Correlação Espaço-Temporal
}

\author{
Diego O. Rodrigues ${ }^{1}$, Leandro Villas ${ }^{1}$ (Orientador)
}

${ }^{1}$ Instituto de Computação, Universidade Estadual de Campinas. Campinas, Brasil Avenida Albert Einstein, 1251 - 13.083-852 - Campinas-SP, Brasil

diegodlrc.ic.unicamp.br, leandrodic.unicamp.br

Resumo. Redes sociais, dispositivos móveis, redes veiculares e de sensores são utilizados como coletores de dados em centros urbanos; dados esses que podem ser utilizados para melhor compreender as dinâmicas das cidades. Muitos estudos tem focado em explorar essas fontes de dados, porém individualmente. Para reduzir o impacto na análise de dados urbanos causada por essa limitação foi desenvolvido o SMAFramework, para realizar a coleta e integração de dados de mobilidade urbana de fontes heterogêneas. Foi proposta uma metodologia para padronizar dados com anotações espaço-temporais provenientes de várias fontes de acordo com um estrutura única de dados (i.e., Grafo Multi-Aspecto ou GMA). A ferramenta apresentada ainda permite que se realize diferentes tarefas de análise de dados. Para avaliar o arcabouço desenvolvido, experimentos foram realizados com dados reais. O primeiro experimento combinou dados de mídias sociais com dados de viagens de táxi a fim de avaliar a correlação espaço temporal entre essas fontes de dados. Em um segundo experimento dados de táxi foram combinados com informações de transito, rotas de transporte público e modelos de experiência de usuário a fim de avaliar como diferentes meios de transporte poderiam ser combinados para melhorar a mobilidade urbana. Ferramentas e metodologias foram desenvolvidas para a execução de cada um desses experimentos, contribuindo com o avanço do estado da arte.

\section{Introdução}

Computação Urbana surge como um tópico em Ciências da Computação que aplica tecnologias de comunicação e informação a fim de melhorar o padrão de vida de cidadãos em centros urbanos (Zheng et al. 2014). Diferentes cidades no mundo planejam utilizar essas tecnologias para melhorar os serviços prestados aos seus cidadãos em áreas como mobilidade, uso de energia, criação de políticas públicas, e outras mais; desse modo tornando factível a construção das, assim denominadas, Cidades Inteligentes. Para criar metodologias de gerenciamento de cidades e satisfazer os novos requisitos dos cidadãos, um passo importante é entender as dinâmicas dessas cidades. Uma forma de obter esse entendimento é por meio da extração de conhecimento de dados gerados na cidade, os quais podem ser coletados de diferentes fontes, como por exemplo, dados gerados por cidadão em seu cotidiano ao utilizar seu telefone móvel ou cartão de crédito.

Abordagens baseadas em dados para planejamento urbano já foram desenvolvidas em teoria e no mundo real. Todavia, boa parte dessas abordagens foca no uso de uma única fonte de dados. Dessa forma, metodologias que exploram múltiplas fontes de dados precisam ser mais estudadas (Ning et al. 2017; Silva et al. 2014). Em particular, a combinação de dados urbanos heterogêneos para obtenção de entendimentos mais precisos sobre a cidade leva a um problema, que é a integração desses dados (Ning et al. 2017; Silva et al. 2014). Esse problema é especialmente crítico para dados espaço-temporais a respeito de fenômenos e eventos. Esses dados geralmente contém coordenadas geográficas, anotações temporais e, possivelmente, identificadores do fenômeno ou evento que foi observado.

Durante o período do mestrado foi desenvolvido um arcabouço, chamado SMAFramework, para lidar com a integração de dados heterogêneos de mobilidade urbana. 
Assim, foram desenvolvidas ferramentas que possibilitem a utilização desses dados, a sua padronização e integração para facilitar a extração de conhecimento. Na dissertação essas ferramentas foram descritas e avaliadas. A primeira avaliação explora a correlação espaço-temporal entre duas fontes de dados distintas. Durante sua realização foi desenvolvido um algoritmo para análise de correlação de amostras no tempo e espaço, chamado Fuzzy Matcher, e um algoritmo para blocagem de uma base de dados espaço-temporal para otimizar a tarefa de percorrer todo o conjunto de dados enquanto são realizadas consultas de vizinhança com características específicas, chamado Bucket Walk. A segunda avaliação buscou explorar dados contextuais para recomendação de rotas híbridas de transporte público e veículos privados de aluguel, como táxis. Nessa segunda avaliação foi desenvolvida uma ferramenta para agrupamento e classificação de fluxos de mobilidade urbana, e também um modelo para avaliação de experiência de usuários em viagens urbanas.

O restante do presente trabalho é organizado como se segue: os trabalhos relacionados são apresentados na Seção 2, a arquitetura do arcabouço é descrita na Seção 3, as principais contribuições obtidas na produção da dissertação são apresentadas na Seção 4, tais contribuições resultaram em publicações em veículos acadêmicos de prestígio, descritos na Seção 5. Os resultados dos experimentos descritos nessas publicações são brevemente listados na Seção 6. Ao fim desse trabalho, na Seção 7, são apresentadas algumas considerações finais e possibilidades futuras para os estudos desenvolvidos durante o período de mestrado.

\section{Trabalhos Relacionados}

Alguns arcabouços para análise de mobilidade têm surgido na literatura. Esses arcabouços não foram criados apenas para mobilidade em cidades, mas também outros cenários, conforme apresentado na Tabela 1. Thakur \& Helmy (Thakur and Helmy 2013) investigam como modelos de mobilidade atuais são adequados para representar mobilidade humana. Patroumpas (Patroumpas 2013) propõem uma metodologia para modelar dados de posições genéricas. Esse modelo introduz um operador de janela deslizante, que permite uma examinação incremental de dados de mobilidade em tempo real. Silva et.al. (Silva et al. 2014) discute o conceito de divisão de camadas de sensoriamento para diferentes tipos de dados disponíveis em uma dada região geográfica. Os autores criam um arcabouço genérico de dados urbanos para análise, porém, é usado um modelo abstrato, que não é específico para nenhum tipo de análise. No presente trabalho, o foco é criar um arcabouço para análise de mobilidade urbana, assim a estrutura de dados é bem definida de forma que todas as camadas de sensoriamento podem ser padronizadas em uma estrutura comum (i.e., a estrutura do GMA). Para realizar análise de tráfego marítimo, Salmin \& Ray (Salmon and Ray 2016) introduzem um arcabouço espaço-temporal capaz de analisar dados em tempo real e arquivados. Os autores exploram também uma especificação de linguagem de consulta padrão, que permite a criação de consultas persistentes e estáticas. Não é apresentado como dados de diferentes fontes poderiam ser utilizados nesse modelo, o que é um fator importante na análise de cenários complexos como a mobilidade urbana.

O SMAFramework, proposto no presente trabalho, é focado na análise de dados gerados nas cidades e observando suas características, como a alta heterogeneidade das fontes de informação, que podem ser disponibilizadas em tempo real, ou estarem armazenadas. Além disso, como o foco desse arcabouço é em mobilidade, é possível explorar detalhes específicos desse contexto que podem facilitar as tarefas de análise. A Tabela 1 apresenta as semelhanças e diferenças entre os arcabouços encontrados na literatura mencionados nessa seção. É possível verificar o número de camadas de sensoriamento que cada arcabouço disponibiliza. Mesmo arcabouços que permitem combinação de fontes heterogêneas podem possuir apenas uma camada independente, isso pois as diferentes 
fontes são combinadas de forma a perder-se a informação sobre a origem dos dados; o que pode não ser ideal em cenário urbano, onde dados de diferentes fontes podem possuir diferentes significados. O SMAFramework é o único que possui uma representação de dados em estrutura unificada de forma a considerar sua origem distinta. Mais detalhes a respeito do arcabouço e o modelo de dados utilizados são descritos na Seção 3.

Tabela 1. Resumo dos trabalhos relacionados enfatizando as diferenças entre outros estudos e o SMAFramework.

$\begin{array}{lccccc} & \begin{array}{c}\text { Dados } \\ \text { Arquivados }\end{array} & \begin{array}{c}\text { Dados } \\ \text { em Tempo Real }\end{array} & \begin{array}{c}\text { Estrutura de Dados } \\ \text { Padronizada }\end{array} & \begin{array}{c}\text { Combinação de } \\ \text { Fontes de Dados }\end{array} & \begin{array}{c}\text { Número de } \\ \text { Camadas } \\ \text { Independentes }\end{array} \\ \begin{array}{l}\text { Thakur \& Helmy } \\ (2013)\end{array} & \mathrm{X} & \mathrm{X} & \mathrm{X} & \mathrm{X} & 1 \\ \begin{array}{l}\text { Patroumpas et. al. } \\ (2013)\end{array} & & \mathrm{X} & & \mathrm{X} & 1 \\ \begin{array}{l}\text { Silva et. al. } \\ (2014)\end{array} & \mathrm{X} & \mathrm{X} & \mathrm{X} & \mathrm{X} & \mathrm{N} \\ \begin{array}{l}\text { Salmon \& Ray } \\ \text { (2016) }\end{array} & \mathrm{X} & \mathrm{X} & \mathrm{X} & \mathrm{X} & 1 \\ \begin{array}{l}\text { SMAFramework } \\ \text { (2017) }\end{array} & \mathrm{X} & \mathrm{X} & \mathrm{X} & \mathrm{N}\end{array}$

\section{SMAFramework}

Esta seção descreve o SMAFramework, arcabouço para análise de dados de mobilidade urbana provenientes de fontes heterogêneas. No topo, dados sobre aspectos urbanos são coletados da cidade por meio de diferentes camadas de sensoriamento (Silva et al. 2014). Esses dados são coletados, por meio de Coletores de Dados, em um formato inicial e salvos em um banco de amostras. Essas amostras serão transformadas, por diferentes operações, no formato determinado para representação dos dados, o Grafo Multi-Aspecto (Kivelä et al. 2014). Essa estrutura de dados permite unificar o padrão das amostras sem perder informações sobre suas diferentes origens. Uma vez nesse formato, abordagens de análise podem ser utilizadas para produção de resultados e extração de conhecimento.

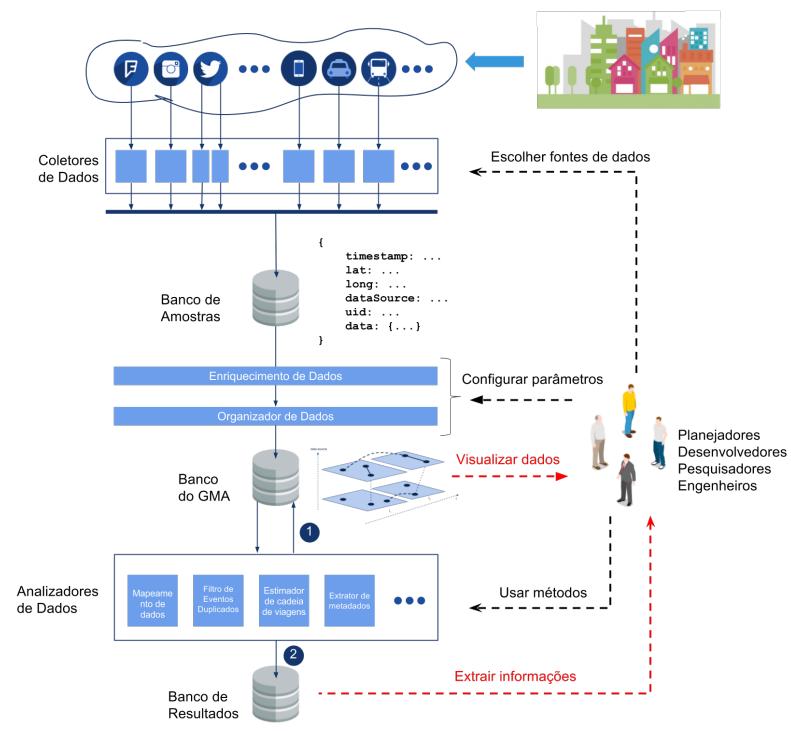

Figura 1. Arquitetura do SMAFramework

O modelo selecionado para representação dos dados dentro do arcabouço é o Grafo Multi-Aspecto. O uso de grafos para representar dados de mobilidade é recorrente na literatura. Essa recorrência, aliada a necessidade de representar alterações ao 
longo do tempo desses dados resultou em diferentes modelos baseados em grafos com suporte a representação do tempo. Os exemplos mais comuns desses modelos são: (i) Imagens Instantâneas (II) (Figueiredo et al. 2011; Tang et al. 2010), onde o grafo é formado por $\mathrm{N}$ grafos desconexos, cada um representando um momento no tempo; (ii) Intervalos de Tempo Contínuos (ITC) (Casteigts et al. 2012), onde as arestas entre os nós são representados como funções que permitem a identificação da existência ou não da aresta dado um momento no tempo; (iii) Aresta Espaço-Temporais (AET) (Nicosia et al. 2013; Kostakos 2009), que usa dois tipos de arestas para representar as interações entre os nós (i.e., arestas espaciais e arestas temporais); (iv) Arestas Espaço-Temporais Mistas (AETM) (Kim and Anderson 2012), similar a abordagem de Arestas Espaço-Temporais, porém, permite a criação de arestas mistas (i.e., arestas espaço-temporais); e finalmente (v) Grafos Multi-Aspecto (GMA) (Wehmuth et al. 2014; Kivelä et al. 2014), que é uma extensão do modelo de grafo que permite a representação de diferentes peculiaridades como distribuição no tempo e origem - dos dados utilizando aspectos. Uma das vantagens de selecionar os GMA para representar os dados das cidades inteligentes é sua capacidade de representação dos diversos tipos de relação presentes nos outros grafos. Além disso, a existência de uma divisão em camadas permite representar os dados e a divisão existem na fonte da qual foram coletados.

A principal função do arcabouço é a extração de conhecimento dos dados, nesse sentido foram desenvolvidas algumas ferramentas que permitam a análise desses dados; essas ferramentas foram adicionadas ao SMAFramework. A produção dessas ferramentas conduziu ao desenvolvimento das principais contribuições do trabalho. Essas contribuições são descritas na Seção 4.

\section{Principais Contribuições}

O arcabouço foi desenvolvido a partir de uma composição agregada das metodologias e algoritmos produzidos durante o mestrado no contexto de análise de dados de mobilidade urbana provenientes de fontes heterogêneas. Além disso, também foi criado um fluxo de tratamento dos dados desde a coleta até a extração de conhecimento. Esses algoritmos e metodologias visam melhorar o desempenho de algumas tarefas essenciais ao uso de dados espaço-temporais, bem como criar novas formas de analisá-los. De forma geral pode-se citar as seguintes contribuições obtidas:

1. Algoritmo para combinar diferentes fontes de dados com anotações espaçotemporais. O algoritmo, denominado Fuzzy Matcher, é uma ferramenta para análise de correlação entre diferentes fontes de dados baseada na posição no tempo-espaço das amostras coletadas. Abordagens semelhantes existem no contexto de Ligação de Registros (i.e., Reccord Linkage), porém em maioria elas exploram dados como endereços e não posições geográficas. A abordagem espaçotemporal mais similar presente na literatura é a ST-LINK (Basik et al. 2018) que considera regiões no espaço - não necessariamente geo-coordenadas. Essa abordagem não fornece informações sobre a qualidade da ligação dos registros, diferente do FuzzyMatcher que fornece pontuações para as ligações. O algoritmo é descrito na Seção 3.2 de (Rodrigues 2018).

2. Algoritmo de blocagem de bases de dados n-dimensionais a fim de melhorar o desempenho de buscas em vizinhança com restrições para máxima distância constante. $\mathrm{O}$ algoritmo, denominado Bucket Walk, permite percorrer conjuntos de dados de forma explorar sua vizinhança com uma menor quantidade de comparações de amostras distantes no espaço-tempo e também de forma paralela. Existem na literatura outros algoritmos para acessar dados espaciais e temporais, como por exemplo o $\mathrm{R}^{*}$-tree (Beckmann et al. 1990). $\mathrm{R}^{*}$-tree é uma 
metologia de indexação de dados que facilita a localização de registros individuais, todavia essa estrutura não apresenta benefícios para a execução de consultas de vizinhança (Sharifzadeh and Shahabi 2010). Outra abordagem, o StreamCube (Feng et al. 2015), organiza seu índice de forma semelhante ao BucketWalk, porém o processo cria o índice sem considerar dados específicos da análise a ser realizada (e.g., distância máxima permitida para as ligações), o que pode resultar em uma redução da performance das consultas de vizinhança. O algoritmo Bucket Walk é descrito na Seção 3.3 de (Rodrigues 2018).

3. Técnica para clusterização e classificação de fluxos de mobilidade. A técnica consiste em três etapas, uma dedicada ao reconhecimento de zonas funcionais no espaço, a partir da técnica de clusterização HDBSCAN (Campello et al. 2015); outra que realiza a contabilização dos fluxos entre as zonas funcionais, e, por fim, uma etapa na qual os fluxos são classificados em principais e secundários. As técnicas de agrupamento de fluxos comumente encontradas na literatura utilizam uma abordagem baseada na divisão de um grid dentro da cidade (Hamedmoghadam-Rafati et al. 2017; Momtazpour and Ramakrishnan 2015), que difere da abordagem proposta, a qual faz uma clusterização das amostras. A técnica é descrita na Seção 3.4 de (Rodrigues 2018).

4. Modelo de avaliação de experiência de usuário em sistemas de transporte urbano. Conjunto de equações que busca medir o tempo percebido pelo usuário ao utilizar meios de transporte urbano, como ônibus, metrôs, andar a pé ou chamar um táxi. O modelo avalia a percepção do usuário em diferentes etapas da viagem de acordo com evidencias achadas em pesquisas realizadas na Europa e Estados Unidos. Além disso, o modelo ainda mede a probabilidade de um usuário em adotar uma rota urbana que contenha um trecho a pé de acordo com o tamanho (em metros) desse trecho. Não se conhece outro modelo semelhante de experiência de usuário no uso de transportes públicos. Esse modelo faz parte da Recomendação de Rotas Híbridas de Transporte Público descrita na Seção 3.5 de (Rodrigues 2018).

É importante destacar que os algoritmos produzidos para todas essas contribuições foram integrados ao SMAFramework, artefato final produzido no processo de elaboração da dissertação. Esse arcabouço possuí código aberto, disponibilizado para a comunidade por meio de repositório no Github ${ }^{1}$. O arcabouço tem como propósito ser uma plataforma para a execução de tarefas relacionadas à análise de dados de mobilidade urbana. Desse modo ele proporciona ferramentas para realizar desde tarefas triviais até resolver desafios relacionados a esse tipo de análise. Diferentes experimentos com dados reais foram realizados a fim de evidenciar o valor do arcabouçou; a apresentação dessas contribuições, e também o resultado dos experimentos realizados foram publicados em veículos acadêmicos de destaque nacional e internacional, listados na Seção 5.

\section{Produção Científica}

Como resultados da produção da dissertação obteve-se publicações em veículos relevantes nacional e internacionalmente. Foram publicados quatro artigos em conferências internacionais, dois em conferências nacionais e um artigo em periódico de alto impacto na área estudada.

\section{- Artigo em Revista Científica Periódica}

- (Rodrigues et al. 2018a): Elsvier Computer Communications (Qualis A2, fator de impacto 2.6)

\footnotetext{
${ }^{1}$ https://github.com/diegopso/smaframework/
} 


\section{- Artigos em Conferências Internacionais}

- (Meneguette et al. 2019): [Aprovado] IEEE International Conference on Communications (Qualis A1, H-index 56)

- (Rodrigues et al. 2018b): IEEE Intelligent Transportation Systems Conference (Qualis B2, H-index 32)

- (Santos et al. 2018): IEEE International Conference on Communications (Qualis A1, H-index 56)

- (Rodrigues et al. 2017): ACM International Conference on Modeling, Analysis and Simulation of Wireless and Mobile Systems (Qualis B1, H-index 18)

\section{- Artigos em Conferências Nacionais}

- (Rodrigues et al. 2018c): SBC Simpósio Brasileiro de Redes de Computadores e Sistemas Distribuídos (Qualis B2)

- (Santos et al. 2017): WGRS/SBRC Workshop de Gerência e Operação de Redes e Serviços do SBRC.

Vale destacar que mais de $50 \%$ dos artigos publicados se encontram no estrato superior do Qualis CAPES (A1, A2 e B1), incluso o artigo publicado no periódico. Para os dois artigos publicados no SBRC, mesmo que não estejam no estrato superior esse é o evento nacional de maior relevância na área de redes de computadores. Além das publicações, ainda foi produzido um arcabouço de código aberto disponibilizado na plataforma GitHub (https://github.com/diegopso/smaframework). Esse arcabouço será utilizado como parte de um minicurso no SBRC'2019. Por fim, o artigo publicado na IEEE ITSC'2018 foi convidado para ser estendido em uma edição especial da IEEE ITS Magazine (fator de impacto 3.0), artigo que está em fase de produção. Os principais resultados publicados nesses trabalhos são apresentados na Seção 6.

\section{Resultados Obtidos}

Os principais resultados obtidos foram: (i) análise de demanda de táxis na cidade de Nova Iorque (Rodrigues et al. 2017); e (ii) análise de composição de diferentes modos de transporte, também em Nova Iorque (Rodrigues et al. 2018b). Na análise de demanda de táxis, apresentada na Figura 2 A e B, interações no Twitter (Figura 2-A) comparadas com dados dos Yellow Taxis por meio do algoritmo Fuzzy Matcher (Figura 2-B) foram usadas para indicar possíveis demandas não atendidas (Rodrigues et al. 2018b); a existência real dessas demandas foi posteriormente analisada com outra base de dados (i.e., Green Taxis) (Rodrigues et al. 2018a). Na análise de composição de modos de transporte, os principais fluxos urbanos foram identificados para diferentes horários no dia e condições de congestionamento (Figura 2-C); em seguida esses fluxos foram usados para avaliar o ganho caso eles tivessem sido percorridos com outros modos de transporte urbano, a avaliação considerava custo, duração e experiência de usuário (Rodrigues et al. 2018b).

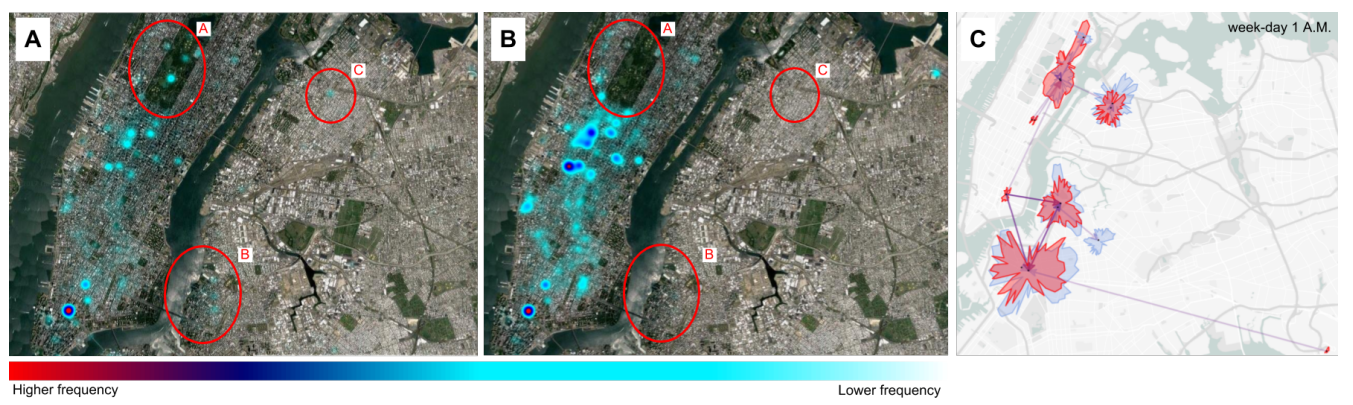

Figura 2. Principais Resultados Obtidos

Além dos resultados apresntados na Figura 2, outros resultados secundários foram obtidos, como: (i) delimitação de regiões de tendência para diferentes camadas de 
sensoriamento (Rodrigues et al. 2018a); (ii) identificação de pares em diferentes fontes de dados que recorrentemente são capturados em lugares diferentes - Seção 4.4 (Rodrigues 2018); e (iii) agrupamento de fluxos urbanos - Seção 4.5 (Rodrigues 2018). Algumas considerações sobre esses resultados, o arcabouço e a dissertação como um todo são apresentados na Seção 7.

\section{Considerações Finais}

O presente texto resume as contribuições produzidas pela dissertação (Rodrigues 2018). $\mathrm{O}$ atual estado da arte em análise de dados urbanos costuma considerar fontes de dados independentes para análise. Todavia, a identificação de padrões em certas dinâmicas urbanas pode não ser possível quando observada uma única fonte de dados. Além disso, muitas das soluções já existentes não contemplam todo o processo de coleta, pré-processamento e extração de informações das bases de dados.

Assim, para atenuar alguns desses problemas em soluções já propostas, foi desenvolvido o arcabouço SMAFramework. Esse arcabouço visa a integração de dados de diferentes fontes a fim de explorar suas complementariedades e permitir visão mais ampla sobre essas dinâmicas. As ferramentas desenvolvidas visam a análise de padrões de mobilidade urbana, e exploram fontes de dados com anotações espaço-temporais. Foram desenvolvidas algumas ferramentas para realizar dois tipos de análises: a primeira focada em visualizar a correlação de duas fontes de dados; e a segunda com foco na avaliação do uso de informações contextuais para recomendação de rotas multi-modais híbridas. As ferramentas desenvolvidas para realizar essas análises contribuem com o avanço do estado da arte em relação aos quatro pontos levantados na Seção 4 e forma divulgados nos veículos descritos na Seção 5. Pretende-se estender os trabalhos com a exploração de dados do clima e modelos de experiência de usuário. Essas extensões estão em fase de produção para o artigo convidado para a IEEE ITS Magazine.

\section{Referências}

[Basik et al. 2018] Basik, F., Gedik, B., Etemoglu, C., and Ferhatosmanoglu, H. (2018). SpatioTemporal Linkage over Location-Enhanced Services. IEEE Transactions on Mobile Computing, 17(2):447-460.

[Beckmann et al. 1990] Beckmann, N., Kriegel, H.-P., Schneider, R., and Seeger, B. (1990). The $\mathrm{R}^{*}$-tree: an efficient and robust access method for points and rectangles. ACM SIGMOD Record, 19(2):322-331.

[Campello et al. 2015] Campello, R. J. G. B., Moulavi, D., Zimek, A., and Sander, J. (2015). Hierarchical Density Estimates for Data Clustering, Visualization, and Outlier Detection. ACM Transactions on Knowledge Discovery from Data, 10(1):1-51.

[Casteigts et al. 2012] Casteigts, A., Flocchini, P., Quattrociocchi, W., and Santoro, N. (2012). Time-Varying Graphs and Dynamic Networks. CoRR, abs/1012.0:20.

[Feng et al. 2015] Feng, W., Zhang, C., Zhang, W., Han, J., Wang, J., Aggarwal, C., and Huang, J. (2015). STREAMCUBE: Hierarchical spatio-temporal hashtag clustering for event exploration over the Twitter stream. Proceedings - International Conference on Data Engineering, 2015May:1561-1572.

[Figueiredo et al. 2011] Figueiredo, D., Nain, P., Ribeiro, B., de Souza e Silva, E., Towsley, D., and Silva, E. D. S. E. (2011). Characterizing Continuous Time Random Walks on Time Varying Graphs. Sigmetrics, cs.SI:1-30.

[Hamedmoghadam-Rafati et al. 2017] Hamedmoghadam-Rafati, H., Steponavice, I., Ramezani, M., and Saberi, M. (2017). A Complex Network Analysis of Macroscopic Structure of Taxi Trips. IFAC-PapersOnLine, 50:9432-9437.

[Kim and Anderson 2012] Kim, H. and Anderson, R. (2012). Temporal node centrality in complex networks. Physical Review E - Statistical, Nonlinear, and Soft Matter Physics, 85(2):1-8.

[Kivelä et al. 2014] Kivelä, M., Arenas, A., Barthelemy, M., Gleeson, J. P., Moreno, Y., and Porter, M. A. (2014). Multilayer networks. Journal of Complex Networks, 2(3):203-271. 
[Kostakos 2009] Kostakos, V. (2009). Temporal graphs. Physica A: Statistical Mechanics and its Applications, 388(6):1007-1023.

[Meneguette et al. 2019] Meneguette, R. I., Rodrigues, D. O., Costa, J. B. D. d., Rosário, D., and Villas, L. A. (2019). A virtual machine migration policy based on multiple attribute decision in vehicular cloud scenario. unpublished.

[Momtazpour and Ramakrishnan 2015] Momtazpour, M. and Ramakrishnan, N. (2015). Characterizing Taxi Flows in New York City. UrbComp, page 9.

[Nicosia et al. 2013] Nicosia, V., Tang, J., Mascolo, C., Musolesi, M., Russo, G., and Latora, V. (2013). Graph Metrics for Temporal Networks, pages 15-40. Springer Berlin Heidelberg.

[Ning et al. 2017] Ning, Z., Xia, F., Ullah, N., Kong, X., and Hu, X. (2017). Vehicular Social Networks : Enabling Smart Mobility. IEEE Communications Magazine, pages 49-55.

[Patroumpas 2013] Patroumpas, K. (2013). Multi-scale window specification over streaming trajectories. Journal of Spatial Information Science, 7(7):45-75.

[Rodrigues 2018] Rodrigues, D. O. (2018). SMAFramework: Arcabouço para Integração de Dados Urbanos Cientes da Correlação Espaço-Temporal. Master's thesis, University of Campinas.

[Rodrigues et al. 2018a] Rodrigues, D. O., Boukerche, A., Silva, T. H., Loureiro, A. A., and Villas, L. A. (2018a). Combining taxi and social media data to explore urban mobility issues. Computer Communications.

[Rodrigues et al. 2017] Rodrigues, D. O., Boukerche, A., Silva, T. H., Loureiro, A. A. F., and Villas, L. A. (2017). SMAFramework: Urban Data Integration Framework for Mobility Analysis in Smart Cities. In Proceedings of the 20th ACM International Conference on Modelling, Analysis and Simulation of Wireless and Mobile Systems, MSWiM '17, pages 227-236, New York, NY, USA. ACM.

[Rodrigues et al. 2018b] Rodrigues, D. O., Fernandes, J. T., Curado, M., and Villas, L. A. (2018b). Hybrid context-aware multimodal routing. In 201821 st International Conference on Intelligent Transportation Systems (ITSC), pages 2250-2255.

[Rodrigues et al. 2018c] Rodrigues, D. O., Silva, T. H., Curado, M., and Loureiro, A. A. F. (2018c). Uso de Dados de Mídias Sociais para Desenvolvimento de Metodologia de Posicionamento de Táxis. In Simpósio Brasileiro de Redes de Computadores e Sistemas Distribuídos.

[Salmon and Ray 2016] Salmon, L. and Ray, C. (2016). Design principles of a stream-based framework for mobility analysis. GeoInformatica, pages 1-25.

[Santos et al. 2018] Santos, F. A., Rodrigues, D. O., Silva, T. H., Loureiro, A. A., Pazzi, R. W., and Villas, L. A. (2018). Context-Aware Vehicle Route Recommendation Platform: Exploring Open and Crowdsourced Data. In IEEE International Conference on Communications.

[Santos et al. 2017] Santos, F. A., Rodrigues, D. O., Silva, T. H., Loureiro, A. A. F., and Villas, L. A. (2017). Rotas Veiculares Cientes de Contexto : Arcabouço e Análise Usando Dados Oficiais e Sensoriados por Usuários sobre Crimes. In Anais do XXII Workshop de Gerência e Operação de Redes e Serviços (WGRS), Belém, Brasil. Sociedade Brasileira de Computação.

[Sharifzadeh and Shahabi 2010] Sharifzadeh, M. and Shahabi, C. (2010). VoR-Tree : R-trees with Voronoi Diagrams for Efficient Processing of Spatial Nearest Neighbor Queries. Proceedings of the 36th International Conference on Very Large Data Bases, 3:1231-1242.

[Silva et al. 2014] Silva, T. H., d. Melo, P. O. S. V., Almeida, J. M., Viana, A. C., Salles, J., and Loureiro, A. A. F. (2014). Participatory sensor networks as sensing layers. In 2014 IEEE Fourth International Conference on Big Data and Cloud Computing, pages 386-393.

[Tang et al. 2010] Tang, J., Scellato, S., Musolesi, M., Mascolo, C., and Latora, V. (2010). Smallworld behavior in time-varying graphs. Physical Review E, 81(5):055101.

[Thakur and Helmy 2013] Thakur, G. S. and Helmy, A. (2013). COBRA: A framework for the analysis of realistic mobility models. In Proceedings - IEEE INFOCOM, pages 3351-3356.

[Wehmuth et al. 2014] Wehmuth, K., Ziviani, A., and Fleury, E. (2014). A Unifying Model for Representing Time-Varying Graphs. Computing Research Repository arXiv.org, I:1-28.

[Zheng et al. 2014] Zheng, Y., Capra, L., Wolfson, O., and Yang, H. (2014). Urban computing: concepts, methodologies, and applications. ACM Transactions on Intelligent Systems and Technology (TIST), 5(3):38. 